\section{Le drame de la mortalité infantile : comment pourrait-on y remédier?}

Domininique Labie
Département de génétique, développement et pathologie moléculaires, Institut Cochin, Inserm U.567, 24, rue du Faubourg Saint-Jacques, 75014 Paris, France.

labie@cochin.inserm.fr

d'allaitement maternel sont autant de facteurs aggravants qui interfèrent évidemment avec la cause directe du décès, qu'elle soit infectieuse ou accidentelle. Ces intrications font apparaître les classifications, dont celle de l'OMS, trop simplificatrices, les maladies pouvant être concomitantes ou survenir séquentiellement: on sait que la rougeole se complique de pneumonie et de diarrhée, que le sida accroît la susceptibilité à d'autres infections, que I'hypotrophie facilite les maladies infectieuses, de même qu'un déficit en vitamine $A$ ou en zinc. Sauf dans quelques-uns des pays étudiés, les bilans épidémiologiques sont souvent incomplets en ce qui concerne les catégories les plus démunies.

Parmi les 10,8 millions de décès recensés, 3,9 (33\%) surviennent dans le premier mois de vie. Cette proportion varie selon les pays entre 20 et $60 \%$, de façon inversement proportionnelle au taux de mortalité global. Une évaluation approximative, et incomplète, attribue $24 \%$ des décès à une étiologie infectieuse, $29 \%$ à une asphyxie, $24 \%$ à la prématurité, $7 \%$ au tétanos. Les autres causes immédiates sont la diarrhée, une pneumonie, le paludisme, la rougeole même non compliquée, $10 \%$ enfin de causes variées ou non déterminées. Quelle que soit la cause du décès, l'hypotrophie en majore l'incidence de façon considérable (Figure 1). Tenant compte de la variabilité observée, les auteurs ont tenté une typologie basée sur les cinq causes majeures de décès, observant ainsi des regroupements géographiques, et ont défini cinq profils épidé- 
miologiques; les taux de décès sont assez voisins lorsqu'il s'agit de diarrhée ou pneumonie, mais extrêmement variables lorsque le paludisme, le sida ou les accidents de la périnatalité sont en cause (Tableau 1). Cette variabilité démontre bien l'importance d'un ciblage national, voire régional, et l'inadéquation d'une évaluation seulement globale. Ces données épidémiologiques ont servi de base à une réflexion sur les modes d'intervention susceptibles de réduire morbidité et mortalité de façon immédiate, par opposition à une action à long terme [4]. Quelles stratégies, quelle efficacité, sachant que les interventions peuvent être préventives ou curatives, et que le prérequis reste l'accessibilité à une population? Les interventions possibles (21 ont été retenues, de l'amélioration des conditions d'hygiène à la délivrance de vraies médications) ont été classées en trois niveaux selon leur efficacité, le niveau 1 définissant une efficacité certaine, celle-ci étant plus limitée pour les niveaux 2 et 3 . Toutes les causes majeures de décès, sauf l'asphyxie néonatale, étaient accessibles à une démarche de niveau 1 et pourraient donc théoriquement être réduites dans un futur proche. Quelques-unes des actions préventives de base sont rappe- lées dans le Tableau // avec leur incidence. La difficulté majeure reste cependant l'accessibilité des populations. Les données de l'UNICEF montrent, en effet, que celle-ci est très variable. Les auteurs ont cherché à évaluer, dans les 42 pays participant à l'étude, quels pourraient être les résultats dans

\begin{tabular}{lccccc}
\hline Profil* & $\mathbf{1}$ & $\mathbf{2}$ & $\mathbf{3}$ & $\mathbf{4}$ & $\mathbf{5}$ \\
Décès (\%) & 109 & 181 & 41 & 169 & 106 \\
Diarrhée(\%) & 24 & 20 & 23 & 17 & $\sim 20$ \\
Pneumonie & 24 & 24 & 15 & 19 & $\sim 20$ \\
Accident de néonatalité & 34 & 26 & 48 & $\sim 20$ & $\sim 25$ \\
Paludisme & $<1$ & 24 & $<1$ & 26 & 6 \\
Sida & $<1$ & $\sim 4$ & $<1$ & $\sim 10$ & 23 \\
Autre & 15 à 20 & $<4$ & 10 à 12 & $<1$ & $\sim 5$ \\
\hline
\end{tabular}

Tableau I. Répartition des dècès d'enfants selon les groupes de pays. * Les chiffres de 1 à 5 correspondent à la classification des pays selon les causes de décès des enfants. Comme l'illustre le tableau, la variation est due essentiellement au paludisme et au sida, l'incidence de ces maladies définissant les groupes (adapté de [3]).

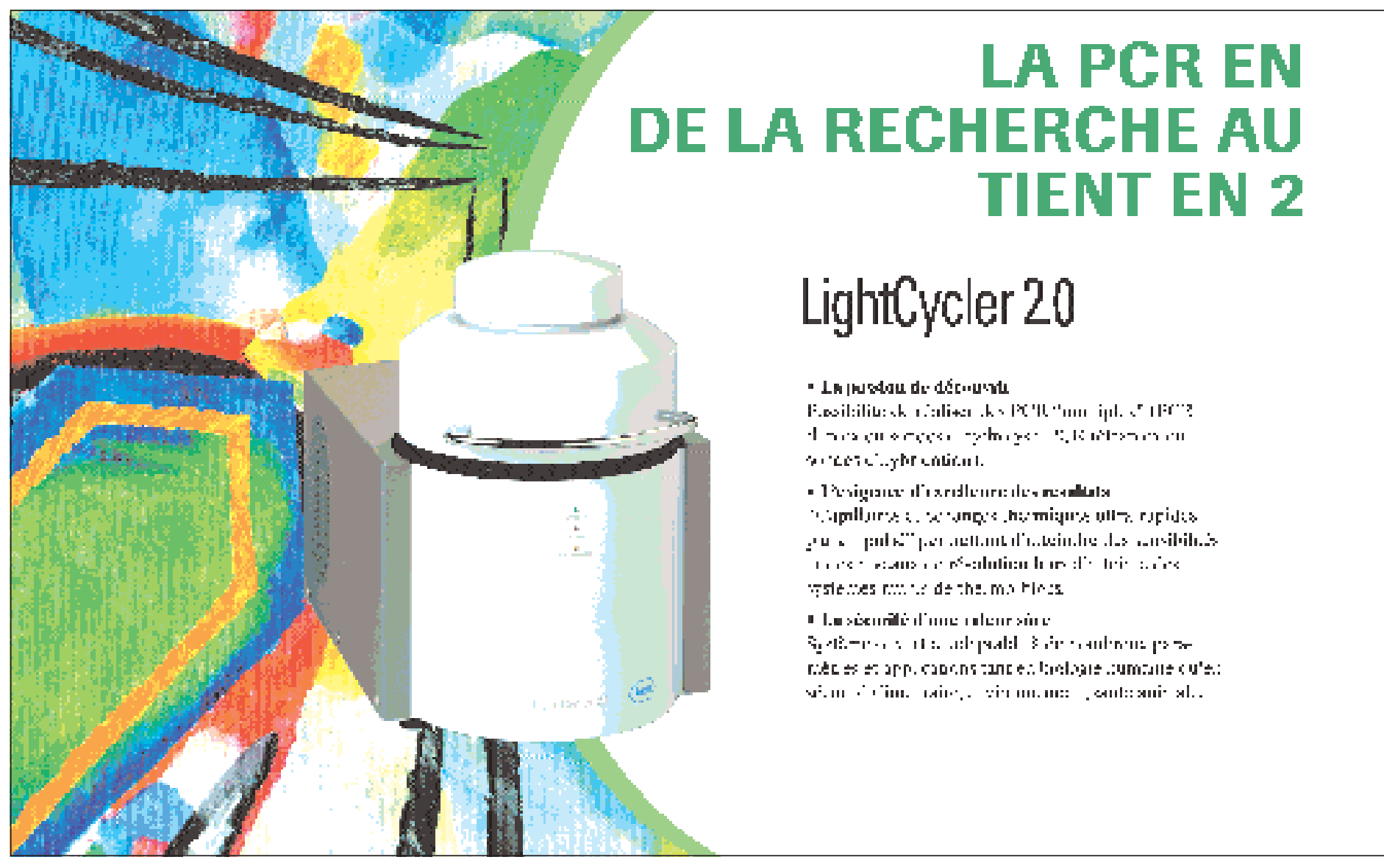


l'éventualité d'une couverture universelle. Ils chiffrent ainsi, pour chacune des interventions simples, la proportion de décès évitables. Une action sur l'alimentation maternelle ou la réhydratation orale pourrait chacune sauver 13 à $15 \%$ des enfants, $5 \%$ environ pourraient l'être par des actions sur le matériel, l'amélioration du traitement anti-paludéen, un complément alimentaire, la prescription d'antibiotiques, un apport prophylactique de zinc. Pourrait-on envisager une couverture universelle polyvalente, c'est-à-dire associant plusieurs de ces actions? Dans ce cas, on évalue à $60 \%$ la proportion de décès

\begin{tabular}{lccc}
\hline Action préventive & $\begin{array}{c}\text { Nombre de décès } \\
\times 10^{3}\end{array}$ & $\begin{array}{c}\text { \% des décès } \\
\text { Allaitement au sein }\end{array}$ & $\begin{array}{c}\text { Taux de couverture } \\
\%\end{array}$ \\
\hline Moustiquaires traitées & 1301 & 13 & 42 à 100 \\
Complément alimentaire & 591 & 7 & faible \\
\hline Supplément en zinc & 459 & 6 & $?$ \\
\hline Hygiène obstétricale & 411 & 5 & $?$ \\
\hline Vaccin anti-Haemophilus & 403 & 4 & 6 à 89 \\
Qualité de l'eau & 326 & 4 & 8 à 98 \\
\hline
\end{tabular}

Tableau II. Estimation du nombre de décès évités par des actions préventives. Le tableau ne liste que les causes principales (adapté de [4]). évités, proportion variable selon le profil épidémiologique, l'efficacité étant d'autant plus faible que prédomine une pathologie périnatale. Un ciblage efficace tiendra toujours compte de la faisabilité; dans un cadre donné, sans matériel externe et à peu de frais, il devrait prévenir au moins un tiers des décès. À ces actions ciblées sur des pathologies définies il faut adjoindre des interventions sur d'autres facteurs comme l'espacement des naissances ou un meilleur accès au système de santé. Même si les données sont encore insuffisantes, les auteurs insistent sur l'importance des résultats obtenus avec des méthodes simples. Ne s'est-on pas focalisé sur des pathologies plus rares et souvent onéreuses? Et que peut-on attendre dans ces conditions des services de santé existants? C'est la question que pose un troisième article [5]. Les avancées scientifiques et technologiques de ces dernières années n'ont eu

\section{TEMPS RÉEL}

DIAGNOSTIC MÉDICAL SYSTËMES

\section{cocorisis: TaciMan \\ 4,2}

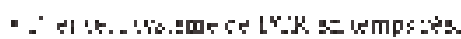

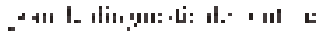

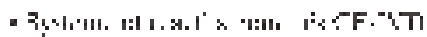

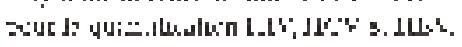

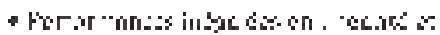

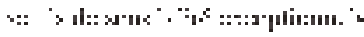

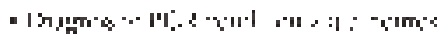

\section{MARQUAGE} CE-IVD

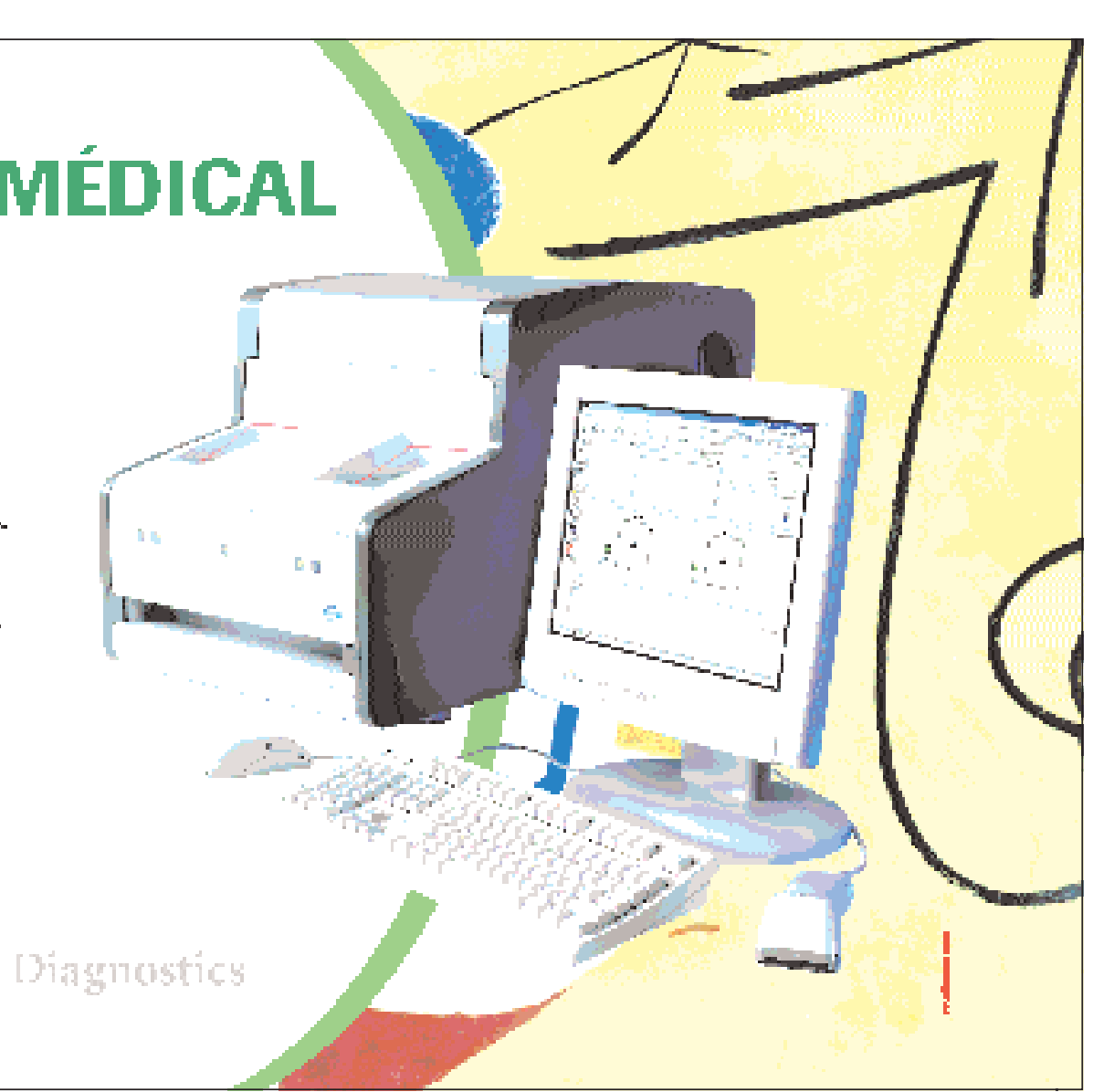


que des résultats très insuffisants, essentiellement parce que leur diffusion n'atteint pas les enfants qui en ont le plus besoin. Quelques expériences ponctuelles réussies démontrent l'importance de la formation du personnel de santé, et de son adaptation à la culture locale. Cinq directions sont suggérées pour améliorer la couverture sanitaire, mais outre la réflexion médicale et scientifique les auteurs insistent sur les études d'évaluation coût/efficacité. Où? Quand? Par qui ?

C'est sur le manque d'équité dans la distribution qu'insiste une nouvelle équipe d'auteurs [6]. II est clair que les différences ne cessent de s'accentuer, non seulement entre pays riches et pays pauvres, mais, à l'intérieur de ces derniers, entre classes aisées et classes démunies. II ne s'agit donc plus d'agir dans un contexte existant, mais de modifier en profondeur certaines stratégies. Ce sont, en effet, les populations les plus pauvres qui souffrent le plus de la pollution de l'eau et de l'air, de l'absence d'équipements sanitaires, de malnutrition. C'est là que les poids de naissance sont insuffisants, les intervalles entre les naissances réduits, les infections fréquentes et la prise en charge aléatoire en cas de maladie. La synergie de ces risques accrus demanderait une couverture plus élevée alors qu'elle est souvent plus faible, que la couverture d'assurance est moindre, le service de santé souvent déficitaire.

Comment réduire ces dysfonctionnements et comment transformer les connaissances en actions [7] ? Diverses options, complémentaires, variables selon les pays, existent, qu'il est impossible de détailler ici. Deux approches ont été suggérées: (1) cibler directement, certaines régions géographiques, certaines minorités ethniques, en améliorant les conditions de vie; (2) opter pour une couverture générale dont on peut espérer qu'elle atteigne aussi des catégories pauvres. Sauf en ce qui concerne le sida, les traitements proposés sont cependant peu onéreux, mais les actions entreprises fragmentées et mal coordonnées. Une analyse exhaustive a révélé lacunes et besoins. Elle devrait permettre d'obtenir dans la décennie qui vient les résultats que demande l'OMS. C'est en fait un SOS qui est lancé à tous. $\diamond$

Child survival : a global health challenge

\section{RÉFÉRENCES}

1. WHO. The world health report 2002: reducing risks, promoting healthy life. Geneva: World Health Organization 2002.

2. Lee JW. Child survival: a global health challenge. Lancet 2003; 362: 262.

3. Black RE, Morris SS, Bryce J. Where and why are 10 million children dying every year? Lancet 2003; 361: 2226-34.

4. Jones G, Steketee RW, Black RE, et al. The Bellago Child Survival Study Group. How many child deaths can we prevent this year? Lancet 2003; 362: 65-71.

5. Bryce J, el Arifeen S, Parlyo G, et al. The multi-country evaluation of $\mathrm{IMCl}$ study group. Reducing child mortality: can public health deliver? Lancet 2003 ; 362 : 159-64.

6. Victora CG, Wagstaff A, Schellenberg JA, et al. Applying an equity lens to child health and mortality: more of the same is not enough. Lancet 2003 ; $362: 233-41$.

7. The Bellago study group on child survival. Knowledge into action for child survival. Lancet 2003 ; $362: 323-7$.

\section{NOUVELLE}

\section{La cible de la warfarine identifiée}

Bernard Le Bonniec
Inserm U.428, UFR des

sciences pharmaceutiques,

4, avenue de l'Observatoire, cofacteur (la protéine S), 75270 Paris Cedex 6, France. qui, synthétisés par les bonniec@pharmacie.univ- hépatocytes, sont tous paris5.fr essentiels pour l'hémo-

stase (Figure 2) [2, 3]. D'autres protéines, dont

> Loin d'être d'origine militaire, le nom de warfarin(e) provient du soutien décisif de la Wisconsin Alumni Research Foundation dans la mise au point d'un médicament pris quotidiennement par des millions de personnes dans le monde. Il s'agit cependant bien du même principe actif que celui contenu dans ce qui est communément appelé la «mort aux rats». La warfarine est un dérivé de la coumarine qui, découverte fortuitement dans les années 1920, cible l'enzyme vitamine $K$ époxyde réductase (VKOR). L'existence de la VKOR était établie depuis 1974, puisque c'est un élément crucial du cycle de la vitamine $K$ (Figure 1), mais ni la protéine ni son gène n'avaient encore été identifiés. Plus qu'un cofacteur, la vitamine $K$ est le cosubstrat d'une $\gamma$-glutamyl carboxylase qui, en la consommant, relâche son époxyde. La quantité de vitamine $K$ apportée par l'alimentation étant limitée, il est impératif de recycler cet époxyde en vitamine $K$ réduite, à nouveau disponible pour la $\gamma$-glutamyl carboxylase, et c'est cette réaction qu'accomplit la VKOR (Figure 1). La fonction de la $\gamma$-glutamyl carboxylase [1] est de transformer en acide $\gamma$-carboxy-glutamique les neuf à douze glutamates du domaine amino-terminal de cinq proenzymes (les facteurs VII, IX, X, la prothrombine, et la protéine $C$ ) et d'un le rôle n'est pas toujours bien défini, subissent cette modification post-traductionnelle : la protéine $Z$ également d'origine hépatique, l'ostéocalcine impliquée dans le métabolisme osseux, la protéine anti-apoptotique Gas6 exprimée par de nombreuses cellules, et deux protéines transmembranaires dites «riches en prolines» exprimées dans la moelle épinière et la thyroïde. La $\gamma$-glutamyl carboxylase reconnaît ses cibles grâce à une séquence particulière portée par le propeptide de toutes les protéines qui subissent cette modification posttraductionnelle et qui sont dites «vitamine $K$ dépendantes». $\varepsilon$ n bloquant la VKOR, la Warfarine interrompt le cycle de 